\title{
New Theoretical Background for Tuberculosis Treatment
}

\author{
Jagadevappa S Patil* \\ V. T's Shivajirao S Jondhle College of Pharmacy, Thane, Maharashtra, India \\ *Corresponding author: Jagadevappa S Patil, V. T's Shivajirao S Jondhle College of Pharmacy, Asangoan, Thane-421 601, Maharashtra, India, E-mail: \\ pharmajspatil@gmail.com
}

Received date: November 06, 2014, Accepted date: November 07, 2014, Published date: November 13, 2014

Copyright: () 2014 Patil JS. This is an open-access article distributed under the terms of the Creative Commons Attribution License, which permits unrestricted use, distribution, and reproduction in any medium, provided the original author and source are credited.

\section{Tuberculosis}

Tuberculosis (TB), a highly contagious chronic bacterial infection, is still a leading killer of young adults worldwide. TB has recently emerged as a major health concern. Each year, approximately 2 million persons worldwide die and 9 million become infected. TB has returned with a new face and the global scourge multidrug resistant TB (MDR$\mathrm{TB})$ is reaching epidemic proportions [1]. The prevalence of TB is continuing to increase because of the increased number of patients infected with human immunodeficiency virus, bacterial resistance to medications, increased international travels and immigration from countries with high prevalence, and the growing numbers of homeless and drug abusers [2]. TB is treated with a multidrug regimen, and is thus exceptionally vulnerable to incidences of side effects, unsatisfactory patient compliances and slow improvement of patients [3].

\section{Etiology of Tuberculosis}

Mycobacteria are aerobic, rod shaped, non-spore forming, and nonmotile bacilli with a waxy coat that causes them to retain the red dye when treated with acid in the acid-fast stains. Mycobacteria typically have a unique cell wall structure crucial to their survival. The welldeveloped cell wall contains a considerable amount of fatty acid, mycolic acid, covalently attached to the underlying peptidoglycanbound polysaccharide arabinogalatan, providing an extraordinary lipid barrier. This barrier is responsible for many of the medically challenging physiological characteristics of $\mathrm{TB}$, including resistance to antibiotics and host defense mechanisms. The composition and quantity of the cell wall components affect the bacteria's virulence and growth rate. The cell wall and its compositions are the key to the survival of mycobacteria, and a more complete understanding of the biosynthetic pathways and gene functions and the development of antibiotics to prevent formation of the cell wall are areas of great interest [4].

\section{Tuberculosis Chemotherapy}

The treatment of TB has witnessed many important changes over the years. With the advent of effective antimycobacterial chemotherapeutic agents in the early 1950s, the two main biological obstacles to successful treatment of TB were the high rate of failure during treatment and the high risk relapse after treatment. The former was due to the selection of drug resistant mutants of $\mathrm{M}$ tuberculosis during the initial stages of treatment when the bacterial populations in the lesions were large. Relapse was due to the re-growth of viable organisms that had persisted in the latent state. To overcome these obstacles it was necessary to give the patients a three-drug combination initially and secondly to continue the treatment for 18 to 24 months with two drugs, and so, long-term treatment with a combination of drugs is required [5]. Treatment of active TB with a single drug should never be attempted, and a single drug should never be added to failing regimen, the result being development of multi drug resistance TB [6]. With the introduction of Rifampicin the outlook for treatment of TB changed dramatically. It was no longer necessary to give streptomycin daily for three months initially to prevent failure. The relapse rate also came down steadily even though the duration of treatment was considerably shorter. This was possible because complete or almost complete sterilization of the lesions became feasible with the introduction of rifampicin and pyrazinamide [7].

\section{New theoretical background}

The current treatment of TB involves systemic therapy with multiple drugs. However, pulmonary infections do not always respond well to such systemic therapy, due to insufficient drug diffusion into pulmonary tissue and lume [8]. Bacteria that have persisted over treatment may develop resistance, but higher drug doses to compensate for poor diffusion may lead to systemic toxicity. Additionally, most patients find it difficult in adhering to treatments that necessitate to several administrations a day to maintain efficient antibiotics concentration. Therefore it is imperative to develop new ways to deliver and use antibiotics to avoid spread of resistance and to improve patient's compliance by decreasing dosing frequency. Antibiotic delivery systems administered through pulmonary route aim to deliver high drug concentrations directly at the site of infection while minimizing systemic biodistribution and toxicity. Rifampicin is a so-called concentration-dependent antibiotic [9]. The rate and extent of bacterial kill is related to the attainment of sufficiently high maximum concentration. Its bacterial activity is directly proportional to concentration at the target site. In recent years, one of the best ways to achieve higher drug levels in the lungs has been the development of new particulate sustained release formulations that are directly delivered to the lungs via pulmonary route. Current literatures available on pulmonary delivery of anti-tubercular agents are focusing only on development of extended release particulate systems using variety of biopolymers. Presently in addition to extended release drug delivery approach an attempt is also being made to include the betacyclodextrin as solubility and permeation enhancer in alveolar epithelium-lining fluids to make the TB treatment more effective.

\section{References}

1. du Toit LC, Pillay V, Danckwerts MP (2006) Tuberculosis chemotherapy: current drug delivery approaches. Resp Res 7:118.

2. Goldrick BA (2004) Once dismissed, still rampant: tuberculosis, the second deadliest infectious disease worldwide. Am J Nurs 104: 68-70. 
3. Prabakaran D, Singh P, Jaganathan KS, Vyas SP (2004) Osmotically regulated asymmetric capsular systems for simultaneous sustained delivery of anti-tubercular drugs. J Control Rel 95: 239-248.

4. Joe M, Bai Y, Nacario RC, Lowary TL (2007) Synthesis of the docosanasaccharide arabinan domain of mycobacterial arabinogalactan and a proposed octadecasaccharide biosynthetic precursor. J Am Chem Soc 129: 9885-9901.

5. Fox W, Ellard GA, Mitchison DA (1999) Studies on the treatment of tuberculosis by the British Medical Research Council tuberculosis units, 1946-1986, with relevant subsequent Publications. Int J Tuberc Lung Dis 3: S231-S279.
6. Iseman MD (1993) Treatment of multidrug-resistant tuberculosis. N Engl J Med 329: 784-791.

7. Jawahar MS (2004) Current trends in chemotherapy of tuberculosis. Indian J Med Res 120: 398-417.

8. Li J, Rayner CR, Nation RL, Oven RJ, Spelman D, et al. (2006) Heteroresistance to colistin in multidrug resistant Acinitobacter baumannii. Antimicrob Agent Chemother 50: 2946-2950.

9. Gumbo T, Louie A, Deziel MR, Liu W, Parsons LM, et al. (2007) Concentration-dependent Mycobacterium tuberculosis killing and prevention of resistance by rifampicin. Antimicrob Agent Chemother 51: 3781-3788. 